\section{International Scientific Journal Theoretical \& Applied Science}

p-ISSN: 2308-4944 (print) e-ISSN: 2409-0085 (online)

Year: $2017 \quad$ Issue: $11 \quad$ Volume: 55

Published: 21.11.2017 http://T-Science.org

SECTION 7. Mechanics and machine construction.
Vladimir Bogutskiy

Associate Professor, Ph.D.,

Sevastopol State University, Russian Federation

bogutskivb@yandex.ru.

Leonid Shron

Associate Professor, Ph.D.,

Sevastopol State University, Russian Federation shronlb@rambler.ru

Eleonora Gordeeva Senior Lecturer,

Sevastopol State University, Russian Federation eleonora.gordeeva@yandex.ru

\title{
SYNTHESIS OF FLEXIBLE MACHINING MODULES FOR REPAIR EQUIPMENT
}

Abstract: The article presents the solution of the problem of synthesizing the structure of a flexible production module for group machining of parts for the conditions of repair production.

Key words: group technological process, automated processing area, subsystem, flexible production module, synthesis structure of the FPM.

Language: Russian

Citation: Bogutskiy V, Shron L, Gordeeva E (2017) SYNTHESIS OF FLEXIBLE MACHINING MODULES FOR REPAIR EQUIPMENT. ISJ Theoretical \& Applied Science, 11 (55): 101-110.

Soi: http://s-o-i.org/1.1/TAS-11-55-13 Doi: crossef https://dx.doi.org/10.15863/TAS.2017.11.55.13

\section{УДК 658.512}

\section{СИНТЕЗ ГИБКИХ МЕХАНООБРАБАТЫВАЮЩИХ МОДУЛЕЙ ДЛЯ УСЛОВИЙ РЕМОНТНОГО ПРОИЗВОДСТВА}

Аннотация: В статье приведено решение задачи синтеза структуры гибкого производственного модуля групповой механической обработки деталей для условий ремонтного производства.

Ключевые слова: групповой технологический процесс, автоматизированный участок обработки, подсистема, гибкий производственный модуль, синтез структуры ГПМ.

\section{Введение}

Анализ литературы $[1,2,3,4,5$ и др.] показывает, что характерным признаком современного производства и в частности ремонтного, является частая сменяемость изделий при росте требований к его производительности. Существующий станочный парк предприятий специализирующихся на производстве и ремонте оборудования содержит зачастую малопроизводительное и морально устаревшее оборудование, не позволяющее оперативно реагировать на решение задач, связанных с изготовлением деталей в процессе ремонта в случае отсутствия запчастей либо невозможности восстановления изношенных деталей. Решением этой проблемы является внедрение в ремонтном производстве автоматизированных участков по механической обработке деталей $[6,7]$.
Для определения рациональной структуры автоматизированных участков механической обработки деталей необходима разработка алгоритма выбора рациональных подсистем и элементов для обработки определенных групп деталей ремонтируемого оборудования $[8,9,10]$.

Как показано в $[11,12]$ в условиях многономенклатурного ремонтного производства целесообразно применять метод группового ремонта деталей, позволяющий свести к минимуму индивидуальные технологические разработки. Этот же принцип заложен в основу технологии переналаживаемого ремонтного производства. При автоматизации процессов механической обработки, как правило, разрабатывают групповой технологический процесс (табл. 1) обработки комплексной детали (рис. 1). 


\section{Материалы и методы исследования}

Рассмотрим процесс синтеза гибкого механообрабатывающего производственного модуля (ГПМ) на примере детали «Шлицевой вал». Анализ группового технологического процесса (табл. 1) механической обработки деталей типа шлицевых валов, показывает необходимость применения определенных типов технологического оборудования, таких как центровально-подрезные и токарные станки, шпоночно-фрезерные станки, сверлильные станки, шлицефрезерные

полуавтоматы, круглошлифовальные станки.

При проектировании автоматизированных участков необходимо формирование множества структур ГПМ с учетом возможности концентрации операций, т. е. применения широкодиапазонного высоконадежного технологического оборудования на базе многооперационных станков с ЧПУ, применения современных конструкций инструментов, технологической оснастки и загрузочных устройств.
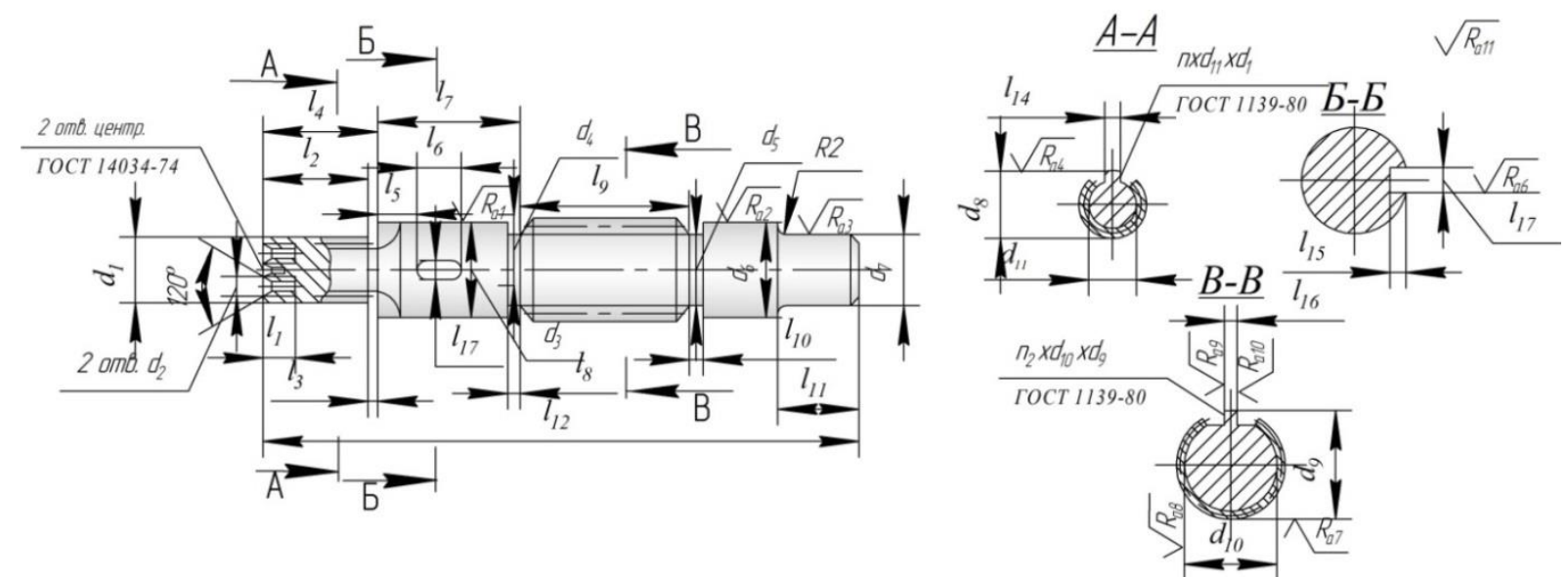

Рисунок 1 - Чертеж комплексной детали «Вал шлицевой».

Таблица 1

\section{Маршрут механической обработки детали (Вал шлицевой)}

\begin{tabular}{|c|c|c|c|}
\hline № операции & Содержание операции & Оборудование & Оснастка \\
\hline 005 & Отрезать заготовку & Фрезерно-отрезной & Тиски \\
\hline 015 & $\begin{array}{l}\text { Фрезеровать торцы в размер } l_{12}, \\
\text { центровать. }\end{array}$ & $\begin{array}{l}\text { Центровально- } \\
\text { подрезной }\end{array}$ & $\begin{array}{l}\text { Приспособление } \\
\text { станке }\end{array}$ \\
\hline 020 & Точить $d_{8}, d_{3}, d_{9}$ предварительно & Токарный с ЧПУ & $\begin{array}{l}\text { Токарный патрон, центр } \\
\text { вращающийся }\end{array}$ \\
\hline 025 & $\begin{array}{l}\text { Точить } d_{7}, d_{6} \text { и } d_{9} \text { предварительно, } \\
\text { точить } d_{7}, d_{6} \text { и } d_{9} \text { начисто, точить } \\
\text { канавку } d_{5} \times l_{10} \text {, снять фаски }\end{array}$ & Токарный с ЧПУ & $\begin{array}{l}\text { Токарный патрон, центр } \\
\text { вращающийся }\end{array}$ \\
\hline 030 & $\begin{array}{lcccc}\text { Точить } & d_{3} & \text { и } & d_{9} & \text { предварительно, } \\
\text { точить } & d_{3} & \text { и } & d_{9} & \text { начисто, точить } \\
\text { канавки } & d_{1} \times & l_{3}, d_{4} \mathrm{x} & l_{8}, \text { снять фаску } \\
\end{array}$ & Токарный с ЧПУ & $\begin{array}{l}\text { Токарный патрон, центр } \\
\text { вращающийся }\end{array}$ \\
\hline 035 & Фрезеровать шпоночный паз $l_{17}$ x $l_{6}$ & Шпоночно- фрезерный & Самоцентрирующие тиски \\
\hline 040 & Обработать $n_{l}$ отверст. $d_{2}$ на $l_{1}$ & Сверлильный & Приспособление УСП \\
\hline 045 & $\begin{array}{l}\text { Фрезеровать } n_{2} \text { шлицев в размер } l_{16} \text {; } \\
\text { до } d_{10}\end{array}$ & $\begin{array}{l}\text { Шлицефрезерный } \\
\text { полуавтомат }\end{array}$ & Центра, поводок \\
\hline 050 & $\begin{array}{l}\text { Фрезеровать } n \text { шлицев в размер } l_{14} \text {; } \\
\text { до } d_{11}\end{array}$ & $\begin{array}{l}\text { Шлицефрезерный } \\
\text { полуавтомат }\end{array}$ & Центра, поводок \\
\hline 055 & Зачистить заусенцы & Верстак & \\
\hline 060 & Шлифовать $d_{8}, d_{3}, d_{9}$ & Круглошлифовальный & Центра, поводок \\
\hline 065 & Шлифовать $d_{7}, d_{6}$ & Круглошлифовальный & Центра, поводок \\
\hline
\end{tabular}

Структурная схема технологической системы такого ГПМ, подвергнутого декомпозиции на подсистемы, показана на рис. 2.При формировании множества структур ГПМ целесообразно применить аппарат морфологического анализа, достаточно полно разработанного для решения подобных технических задач, в частности проектирования 
станков, их узлов и механизмов, способов формообразования и т.д. [7, 8, 9]. Также морфологический анализ может быть использован в качестве аппарата для формирования множества структур ГПМ и оценки их вариантов по укрупненным показателям. Для решения поставленной задачи построена декомпозиционная схема (см. табл. 2), данные для которой выбирались на основании структурной схемы, приведенной на рис. 2, подробно анализируя признаки и характеристики подсистем и элементов, а также выявляя связи между ними и характеристик деталей, на основе которых разрабатывалась комплексная деталь (см. рис. 1).

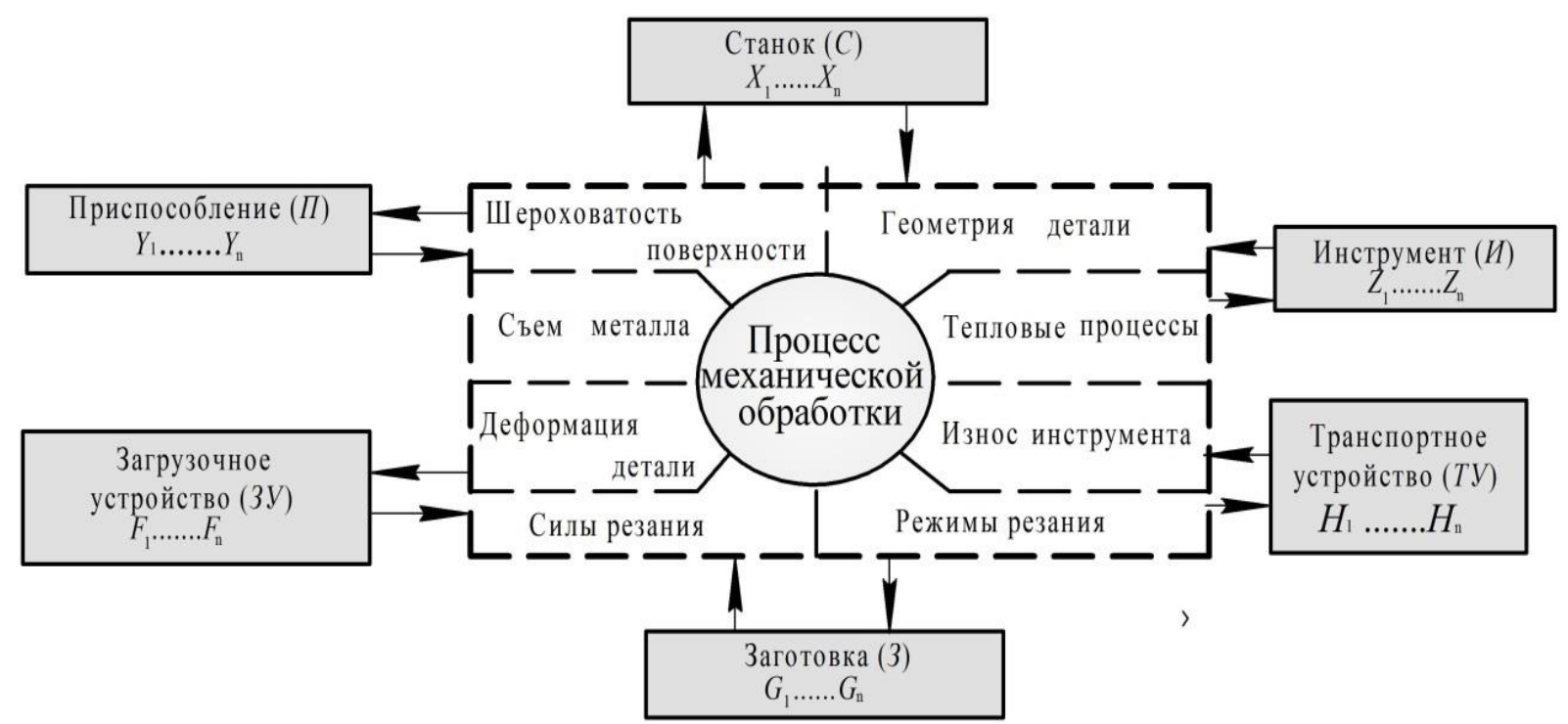

Рис. 2. Структурная схема технологической системы ГПМ

Таблица 2

Декомпозиционная схема анализа задачи формирования структуры ГПМ механической обработки деталей типа тел вращения на примере шлицевого вала

\begin{tabular}{|c|c|c|c|c|c|}
\hline Признак подсистемы & \multicolumn{5}{|c|}{ Вариант подсистемы } \\
\hline 1 & \multicolumn{5}{|c|}{2} \\
\hline \multicolumn{6}{|c|}{ Подсистема ГПМ - Станок $(C)$} \\
\hline \multirow{2}{*}{$\begin{array}{l}\text { 1. Центровально- } \\
\text { подрезной }-X_{I}\end{array}$} & $X_{11}$ & \begin{tabular}{l|l}
$X_{12}$ \\
\end{tabular} & \multicolumn{2}{|l|}{$X_{13}$} & $X_{14}$ \\
\hline & $\mathrm{MP}-179$ & 2A911-1 & \multicolumn{2}{|c|}{ EM535M } & $2 \Gamma 942.18$ \\
\hline \multirow{6}{*}{ 2. Токарный с ЧПУ - $X_{2}$} & \multicolumn{2}{|c|}{$X_{21}$} & \multicolumn{3}{|c|}{$X_{22}$} \\
\hline & \multicolumn{2}{|c|}{$\begin{array}{c}\text { Автоматы и полуавтоматы } \\
\text { (11Б16ВФ4, 11Б40ПФ4) }\end{array}$} & \multicolumn{3}{|c|}{$\begin{array}{c}\text { Токарно - револьверные (1П420ПФ40, } \\
\text { 1В340Ф30 1325Ф30) }\end{array}$} \\
\hline & \multicolumn{2}{|c|}{$X_{23}$} & \multicolumn{3}{|c|}{$X_{24}$} \\
\hline & \multicolumn{2}{|c|}{$\begin{array}{c}\text { Патронно - центровые } \\
(1757 \Phi 3,1757 \Phi 4,16 Р Т 30 Р \Phi 3)\end{array}$} & \multicolumn{3}{|c|}{$\begin{array}{c}\text { Автоматы для РТК и ГПМ (РТ777Ф3, } \\
\text { 1720ПФ30, 1740РФ3) }\end{array}$} \\
\hline & \multicolumn{5}{|c|}{$X_{25}$} \\
\hline & \multicolumn{5}{|c|}{ Токарные обрабатывающие центры (1C740, МТ 540, АC16М25Ф4) } \\
\hline \multirow{2}{*}{$\begin{array}{l}\text { 3. Шпоночно - фрезерный } \\
-X_{3} \\
\end{array}$} & \multicolumn{2}{|c|}{$X_{31}$} & \multicolumn{3}{|c|}{$X_{32}$} \\
\hline & \multicolumn{2}{|c|}{ 6ДМ83ШФ2, FV-200 } & \multicolumn{3}{|c|}{$692 \mathrm{M}, 6930$} \\
\hline \multirow{2}{*}{ 4. Сверлильные $-X_{4}$} & $X_{41}$ & $X_{42}$ & \multirow{2}{*}{\multicolumn{2}{|c|}{$\frac{X_{43}}{2 \mathrm{H} 135 \Phi 2}$}} & $X_{44}$ \\
\hline & 2P118Ф2 & $400 \mathrm{~V}$ & & & $2 \mathrm{H} 135 \Phi 2$ \\
\hline \multirow{2}{*}{ 5. Шлицефрезерный - $X_{5}$} & \multicolumn{2}{|c|}{$X_{51}$} & \multicolumn{3}{|c|}{$X_{52}$} \\
\hline & \multicolumn{2}{|c|}{ 5А352ПФ2, ВСH-620 NC22, CNC25 } & \multicolumn{3}{|c|}{$5350,5350 \mathrm{~B}$} \\
\hline \multirow{2}{*}{$\begin{array}{l}\text { 6. Круглошлифовальный с } \\
\text { ЧПУ }-X_{6} \\
\end{array}$} & \multicolumn{2}{|c|}{$X_{61}$} & \multicolumn{3}{|c|}{$X_{62}$} \\
\hline & 3B1 & {$[-400.2$} & & 1510 & I120CФ3 \\
\hline & Подсистел & Приспособлен & ие $(\Pi)$ & & \\
\hline 1 Токапные патпоны - $Y_{\text {}}$ & $Y_{11}$ & & 12 & & $Y_{13}$ \\
\hline 1. гокарные патроны - 1 & Трехкулачковь & Быстроперег & алаживаемы & Бы & реналаживаем \\
\hline
\end{tabular}




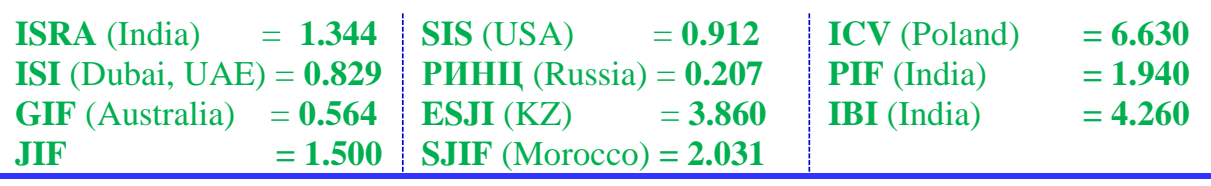

\begin{tabular}{|c|c|c|c|c|c|c|c|}
\hline \multirow[t]{6}{*}{1} & \multicolumn{7}{|c|}{2} \\
\hline & механизированные & \multicolumn{4}{|c|}{ е клиновые } & \multicolumn{2}{|c|}{ ые реечные } \\
\hline & \multicolumn{2}{|c|}{$Y_{14}$} & \multicolumn{3}{|c|}{ 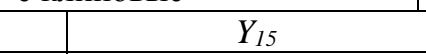 } & \multicolumn{2}{|c|}{$Y_{16}$} \\
\hline & \multicolumn{3}{|c|}{$\begin{array}{l}\text { Комбинированные } \\
\text { универсально - наладочные }\end{array}$} & \multicolumn{2}{|c|}{ Пневматические } & \multicolumn{2}{|c|}{ Гидравлические } \\
\hline & \multicolumn{3}{|c|}{$Y_{17}$} & \multicolumn{2}{|c|}{$Y_{18}$} & \multicolumn{2}{|r|}{$Y_{110}$} \\
\hline & \multicolumn{3}{|c|}{ Электромеханические } & \multicolumn{2}{|c|}{ Поводковые } & \multicolumn{2}{|r|}{ Цанговые } \\
\hline \multirow{4}{*}{$\begin{array}{c}\text { 2. Фрезерные } \\
\text { приспособления - } Y_{2}\end{array}$} & $Y_{21}$ & & & \multicolumn{2}{|c|}{$Y_{22}$} & \multicolumn{2}{|r|}{$Y_{23}$} \\
\hline & \multicolumn{3}{|c|}{$\begin{array}{c}\text { Тиски } \\
\text { пневмогидравлические }\end{array}$} & \multicolumn{2}{|c|}{ Тиски пневматические } & \multicolumn{2}{|r|}{$\begin{array}{l}\text { Поворотно-- } \\
\text { делительные }\end{array}$} \\
\hline & $Y_{24}$ & & & & $Y_{25}$ & & $Y_{26}$ \\
\hline & $\begin{array}{r}\text { Приспособлє } \\
\text { самоценриру } \\
\text { зажимог } \\
\end{array}$ & $\begin{array}{l}\text { e c } \\
\text { (им }\end{array}$ & & $\begin{array}{r}\mathrm{Y}_{\mathrm{H}} \\
\mathrm{c} \\
\text { шात }\end{array}$ & $\begin{array}{l}\text { осально - } \\
\text { чное для } \\
\text { ных пазов }\end{array}$ & & $\begin{array}{c}\text { ниверсально - } \\
\text { сборочное для } \\
\text { сверления }\end{array}$ \\
\hline & \begin{tabular}{l|}
$Y_{31}$ \\
\end{tabular} & & $Y_{32}$ & & $Y_{33}$ & & $Y_{34}$ \\
\hline 3. Шлифовальные - $Y_{3}$ & $\begin{array}{c}\text { Поводковые } \\
\text { патроны }\end{array}$ & & $\begin{array}{l}\text { нтов } \\
\text { нути }\end{array}$ & & $\begin{array}{r}\text { ДвухповоротІ } \\
\text { хомутики } \\
\end{array}$ & ные & $\begin{array}{c}\text { Самозажимные } \\
\text { патроны } \\
\end{array}$ \\
\hline & Подсистем & $\Pi \mathrm{M}$ & $-И_{H}$ & струме & & & \\
\hline & $Z_{11}$ & & & & & & $Z_{13}$ \\
\hline & $\begin{array}{l}\text { Резцы для нар } \\
\text { основных поверх }\end{array}$ & $\begin{array}{l}\text { ных } \\
\text { тей }\end{array}$ & & $\begin{array}{l}\text { Зцы д } \\
\text { полнит } \\
\text { верхно } \\
\end{array}$ & $\begin{array}{l}\text { наружных } \\
\text { ых }\end{array}$ & $\begin{array}{l}\text { РезL } \\
\text { осно }\end{array}$ & $\begin{array}{l}\text { расточные для } \\
\text { ных поверхностей }\end{array}$ \\
\hline 1. Инструмент токарный - & $Z_{14}$ & & & $Z_{15}$ & $Z_{16}$ & & $Z_{17}$ \\
\hline$Z_{1}$ & $\begin{array}{l}\text { Резцы расточнь } \\
\text { дополнительных } \\
\text { поверхностей }\end{array}$ & для & & Сверл & Зенке & ры & Развертки \\
\hline & $Z_{18}$ & & & & & & $Z_{20}$ \\
\hline & Метчики & & & Фрезь & нцевые & & езы шпоночные \\
\hline & $Z_{21}$ & & & & & & $Z_{23}$ \\
\hline & $\begin{array}{c}\text { Комбинирован } \\
\text { сверла }\end{array}$ & & & Центров & ые сверла & & иральные сверла \\
\hline & $Z_{24}$ & & & & & & $Z_{26}$ \\
\hline 2. Инструмент сверлильно & Зенкеры & & & Разверт & востовые & & вертки насадные \\
\hline - фрезерно - расточной - & $Z_{27}$ & & & & & & $Z_{29}$ \\
\hline$Z_{2}$ & Зенковки кониче & & Зен & ковки ц & ндрические & & етчики машинные \\
\hline & $Z_{210}$ & & & & & & $Z_{212}$ \\
\hline & Расточные ре & & & Фpe31 & нцевые & & езы шпоночные \\
\hline & $Z_{213}$ & & & & & & $Z_{215}$ \\
\hline & Фрезы дисков & & & Фрезі & рцевые & & резы червячные \\
\hline 3. Инструмент & $Z_{31}$ & & $Z$ & 32 & $Z_{33}$ & & \begin{tabular}{|l|}
$Z_{34}$ \\
\end{tabular} \\
\hline шлифовальный $-Z_{3}$ & Круги ПП & & оуги & ПВД & Круги ПВ & & Круги ПВК \\
\hline & Подсистема ГПМ & Загру & 304 & Hое устा & тво $(3 У)$ & & \\
\hline 1. Промышленные роботы & $F_{11}$ & & $F$ & 12 & $F_{13}$ & & $F_{14}$ \\
\hline$-F_{1}$ & Портальные & & апол & Іьные & Подвеснь & & На станке \\
\hline & $F_{21}$ & & & & & & $F_{23}$ \\
\hline 2. Магазины - накопители & Прямолинейн & & & & Іьные & & Трубчатые \\
\hline$-F_{2}$ & $F_{24}$ & & & & & & $F_{26}$ \\
\hline & Штыревые & & & & говые & & Бункерные \\
\hline & Подсистема ГПМ & ранс & порл & гное уст & ство $($ ТУ) & & \\
\hline & & & & & & & 12 \\
\hline & Тактог & стол & & & Конвей & еры & о спутниками \\
\hline & & & & & & & 14 \\
\hline 1. Транспортеры $-H_{l}$ & Транспорте & нап & льнн & ые & Трансг & горте & ы подвесные \\
\hline & & & & & & & 16 \\
\hline & $\begin{array}{r}\text { Рельсовые } \\
\text { p }\end{array}$ & $\begin{array}{l}\text { Інспс } \\
\text { ты }\end{array}$ & ртн & & $\begin{array}{r}\text { Моноре } \\
\text { тран }\end{array}$ & $\begin{array}{l}\text { лльсо } \\
\text { спор }\end{array}$ & $\begin{array}{l}\text { ые подвесные } \\
\text { дые роботы }\end{array}$ \\
\hline
\end{tabular}




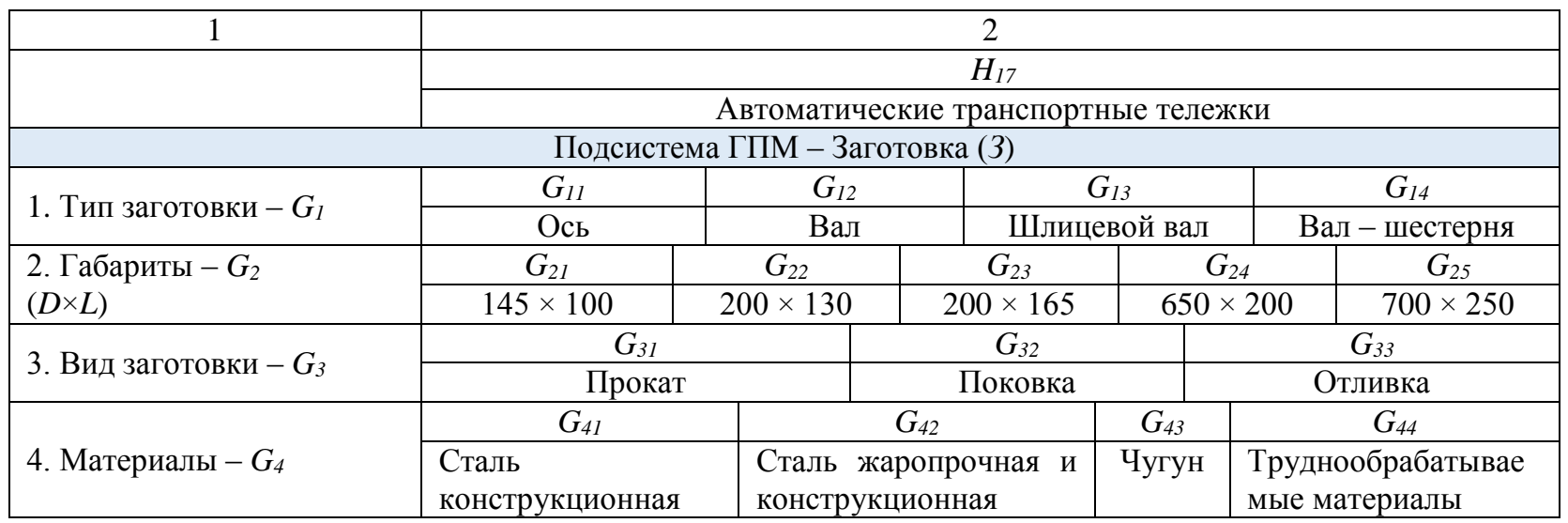

Построенная декомпозиционная схема - это $n$-мерное поисковое пространство, $R^{n}=\left\{X_{\xi}\right\}$, в котором каждое решение представлено вектором $x \in R^{n}$, являющимся множеством из набора альтернатив $X_{\xi}^{\beta}$ вида

$$
X=\left\{X_{1}^{\beta}, \ldots X_{i}^{\beta}, \ldots X_{n}^{\beta}\right\}
$$

После построения декомпозиционной схемы перейдем к синтезу вариантов структуры ГПМ. Из множества признаков $(n=1,2, \ldots, N)$ подсистемы «станок» $(C)$, который может быть центровально-подрезным шлицефрезерным, токарным, шпоночно-фрезерным, сверлильным, круглошлифовальным, выделим наиболее приемлемые для заданных условий. При синтезе структурных вариантов участков применимы различные типы перечисленных станков, которые обеспечат выполнение технологического процесса, но имеют различные характеристики (надежность, принципы действия, габаритные размеры, масса, точность, стоимость).

Пусть имеется $s$ типов $n$-го станка, $(s-$ порядковый номер типа данного станка; $s=1,2, \ldots, S)$. Обозначив через $X_{N S}$ совокупность параметров $n$-го станка $s$-типа, множество состояний подсистемы «С» представим в виде матрицы:

$$
\|C\|=\left\|\begin{array}{cccc}
X_{11} & X_{21} & \ldots & X_{N 1} \\
X_{12} & X_{22} & \ldots & X_{N 2} \\
\ldots & \ldots & \ldots & \ldots \\
X_{1 S_{1}} & X_{2 S_{2}} & \ldots & X_{N S_{N}}
\end{array}\right\| .
$$

Совокупность параметров $m$ - го вида $w$ - го типа подсистемы «приспособление» (П) обозначим через $Y_{M N}$. Тогда множество состояний этой подсистемы запишем как

$$
\|\Pi\|=\left\|\begin{array}{cccc}
Y_{11} & Y_{21} & \ldots & Y_{M 1} \\
Y_{12} & Y_{22} & \ldots & Y_{M 2} \\
\ldots & \ldots & \ldots & \ldots \\
Y_{1 W_{1}} & Y_{2 W_{2}} & \ldots & Y_{M W_{N}}
\end{array}\right\| .
$$

где $m=1,2, \ldots, M ; w=1,2, \ldots, W$.

Множество состояний подсистемы «инструмент» $(И)$ представим как $(i=1,2, \ldots I-$ порядковый номер признака; $r=1,2, \ldots . R$ - номер варианта признака):

$$
\|h\|=\left\|\begin{array}{cccc}
Z_{11} & Z_{21} & \ldots & Z_{J 1} \\
Z_{12} & Z_{22} & \ldots & Z_{J 2} \\
\ldots & \ldots & \ldots & \ldots \\
Z_{1 R_{1}} & Z_{2 R_{2}} & \ldots & Z_{I R_{i}}
\end{array}\right\| .
$$

Свойства подсистемы «загрузочное устройство» (ЗУ) задаем множеством состояний $F_{k t}(k=1,2, \ldots, K ; t=1,2, \ldots, T)$ и представим матрицей:

$$
\|3 Y\|=\left\|\begin{array}{cccc}
F_{11} & F_{21} & \ldots & F_{K 1} \\
F_{12} & F_{22} & \ldots & F_{K 2} \\
\ldots & \ldots & \ldots & \ldots \\
F_{1 T_{1}} & F_{2 T_{2}} & \ldots & F_{K T_{p}}
\end{array}\right\| .
$$

По аналогии также выражаем множество состояний подсистемы «транспортное устройство» $(T У)(p=1,2, \ldots, P$ - порядковый номер признака; $q=1,2, \ldots, Q$ - номер варианта признака):

$$
\|T Y\|=\left\|\begin{array}{cccc}
H_{11} & H_{21} & \ldots & H_{P 1} \\
H_{12} & H_{22} & \ldots & H_{P 2} \\
\ldots & \ldots & \ldots & \ldots \\
H_{1 Q_{1}} & H_{2 Q_{2}} & \ldots & H_{P Q_{p}}
\end{array}\right\| .
$$

Свойства технологического элемента «заготовка» (3) задаем множеством состояний $G_{l u}$ $(1=1,2, \ldots, L ; u=1,2, \ldots, U)$ представим как 


\begin{tabular}{|c|c|c|c|c|c|c|}
\hline Impact Factor: & $\begin{array}{l}\text { ISRA (India) } \\
\text { ISI (Dubai, UAE } \\
\text { GIF (Australia) } \\
\text { JIF }\end{array}$ & $\begin{array}{l}=1.344 \\
=0.829 \\
=0.564 \\
=1.500\end{array}$ & $\begin{array}{l}\text { SIS (USA) } \\
\text { PИНЦ (Russia) } \\
\text { ESJI (KZ) } \\
\text { SJIF (Morocco) }\end{array}$ & $\begin{array}{l}=0.912 \\
=0.207 \\
=\mathbf{3 . 8 6 0} \\
=\mathbf{2 . 0 3 1}\end{array}$ & $\begin{array}{l}\text { ICV (Poland) } \\
\text { PIF (India) } \\
\text { IBI (India) }\end{array}$ & $\begin{array}{l}=6.630 \\
=1.940 \\
=4.260\end{array}$ \\
\hline
\end{tabular}

$$
\|3\|=\left\|\begin{array}{cccc}
G_{11} & G_{21} & \ldots & G_{L 1} \\
G_{12} & G_{22} & \ldots & G_{L 2} \\
\ldots & \ldots & \ldots & \ldots \\
G_{1 U_{1}} & G_{2 U_{2}} & \ldots & G_{L U_{l}}
\end{array}\right\| .
$$

Обозначение и расшифровка параметров $X_{n s}$, $Y_{m w}, Z_{i r}, F_{k t}, H_{p q}, G_{l u}$ ГПМ приведены в табл. 2.

Варианты подсистем выбираем из табл. 2 в качестве основы для построения графа (рис. 3), характеризующего множество возможных структур автоматизированных участков, которое равно числу путей в графе.

С учетом большого количества вариантов синтез решения осуществляем, начиная с выбора вариантов основных подсистем, включая все более и более существенные признаки. Кроме того, следует руководствоваться списком технических требований, определяющихся в первую очередь свойствами такой подсистемы, как заготовка.

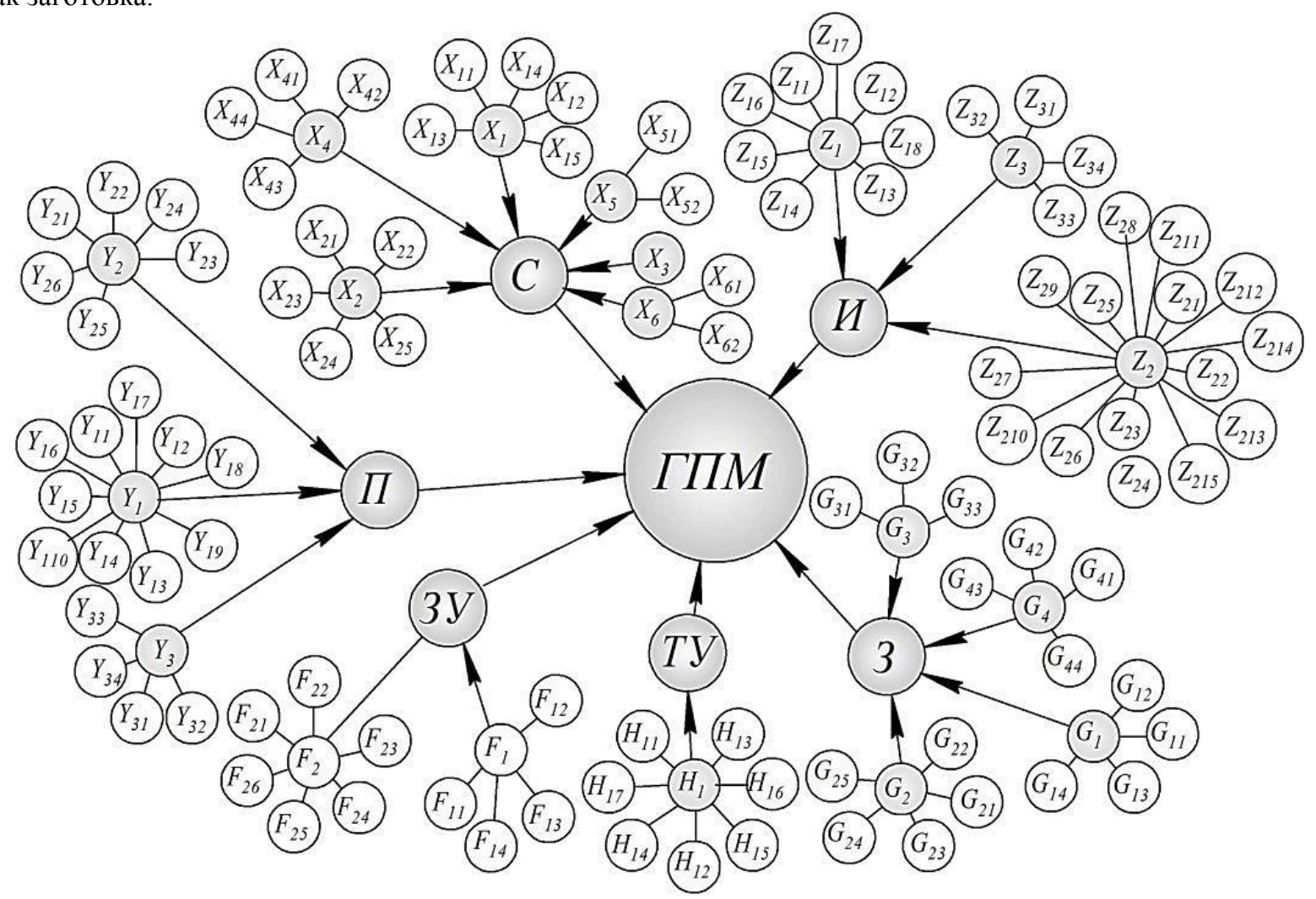

Рисунок 3 - Граф формирования ГПМ

Такими требованиями для подсистемы «С» являются: количество выполняемых операций (многооперационный $-K_{1}^{\prime}=1,0 ; \quad$ двух операционный $-K_{1}^{\prime}=0,75$; однооперационный $\left.K_{1}^{\prime}=0,5\right) ; \quad$ способ смены инструмента (автоматический $-K_{2}^{\prime}=2,0$; механизированный $K_{2}^{\prime}=0,75 ;$ ручной $-K_{2}^{\prime}=0,5$ ); система управления
Например, описание заготовки с конкретными ее свойствами с учетом диапазона изменяющихся параметров в условиях ремонтного производства выглядит как:

1. Тип заготовки - шлицевой вал $-G_{13}$;

2. Габариты $(D \times L) 200 \times 165-G_{23}$;

3 . Вид заготовки - прокат $-G_{31}$;

4. Материал - сталь конструкционная $-G_{41}$.

Выбор вариантов структур ГПМ осуществляем по укрупненным показателям в соответствии с перечнем требований, предьявленных к подсистемам с учетом их основных функций, а также качественных показателей, характеризующих возможность подсистемы адаптироваться к изменению номенклатуры обрабатываемых деталей и к различным производственным ситуациям. (микропроцессорная система ЧПУ $-K_{3}^{\prime}=1,0$; аппаратная система УПУ $-K_{3}^{\prime}=0,75$; цикловая система ПУ $\left.-K_{3}^{\prime}=0,5\right)$.

Суммарный качественный показатель для каждой из возможных структур определяем по формуле 


\begin{tabular}{|c|c|c|c|c|c|c|}
\hline Impact Factor: & $\begin{array}{l}\text { ISRA (India) } \\
\text { ISI (Dubai, UAF } \\
\text { GIF (Australia) } \\
\text { JIF }\end{array}$ & $\begin{array}{l}=1.344 \\
=0.829 \\
=0.564 \\
=1.500\end{array}$ & $\begin{array}{l}\text { SIS (USA) } \\
\text { PИНЦ (Russia) } \\
\text { ESJI (KZ) } \\
\text { SJIF (Morocco) }\end{array}$ & $\begin{array}{l}=\mathbf{0 . 9 1 2} \\
=\mathbf{0 . 2 0 7} \\
=\mathbf{3 . 8 6 0} \\
=\mathbf{2 . 0 3 1}\end{array}$ & $\begin{array}{l}\text { ICV (Poland) } \\
\text { PIF (India) } \\
\text { IBI (India) }\end{array}$ & $\begin{array}{l}=6.630 \\
=1.940 \\
=4.260\end{array}$ \\
\hline
\end{tabular}

$$
K_{c}=1-\sum_{i=1}^{n} \frac{1-K_{i}^{\prime}}{n^{\prime}},
$$

Для структурного варианта участка формула принимает вид:

$$
K_{c y}=\left[1-\sum_{i=1}^{n^{\prime}} \frac{1-K_{i}^{\prime}}{n^{\prime}}\right] N
$$

где $N$ - число станков на участке.

Наибольшие численные значения $K_{c y}$ принадлежат следующим структурным вариантам:

$$
\begin{gathered}
C_{1}=X_{14} \Rightarrow X_{22} \Rightarrow X_{32} \Rightarrow X_{44} \Rightarrow X_{51} \Rightarrow X_{62} \\
C_{2}=X_{14} \Rightarrow X_{25} \Rightarrow X_{51} \Rightarrow X_{62}
\end{gathered}
$$

Вариант $C_{l}$ имеет численное значение $K_{C y l}=$ 0,65 , вариант $C_{2}-K_{C V 2}=0,68$. Выбранные варианты содержат станки, позволяющие создать на их основе автоматизированный участок, причем в первом варианте $\left(C_{l}\right)$ используется большее количество станочного оборудования (2Г942.18, 1В340Ф30; 692M; 2Н135Ф2; 5АЗ52ПФ2; 3М151Ф2) - шесть станков, а во втором варианте $\left(C_{2}\right)$ - четыре единицы оборудования $\quad$ (2Г942.18; 5АЗ52ПФ2; ЛШ120СФ3) за счет более широких технологических возможностей токарного обрабатывающего центра АC16M25Ф4, способного выполнять кроме необходимого перечня токарных операций, также фрезерование продольных пазов и нецентровое сверление и др.

Качественные

показатели

$K_{i}^{\prime \prime}$

приспособления выбирали в соответствии со следующими техническими требованиями: способ загрузки и базирования (автоматический $K_{1}^{\prime \prime}=1,0$; механизированный $-K_{1}^{\prime \prime}=0,75$; ручной $\left.K_{1}^{\prime \prime}=0,5\right)$; позиционирование (автоматическое $K_{2}^{\prime \prime}=1,0$; механизированное $-K_{2}^{\prime \prime}=0,75$; ручное $\left.K_{2}^{\prime \prime}=0,5\right)$; Возможность реализации на базе унифицированных комплектов (полностью $-K_{3}^{\prime \prime}$ $=1,0$; частично $-K_{3}^{\prime \prime}=0,75 ;$ нет $\left.-K_{3}^{\prime \prime}=0,5\right)$.

По аналогии с предыдущим поиском рациональных вариантов подсистем определим качественные суммарные показатели для подсистемы «П», наибольшую величину которых имеют:

$$
\begin{gathered}
\Pi_{1}=Y_{22} \Rightarrow Y_{14} \Rightarrow Y_{23} \Rightarrow Y_{26} \Rightarrow Y_{24} \Rightarrow Y_{34} ; \\
\Pi_{2}=Y_{22} \Rightarrow Y_{14} \Rightarrow Y_{24} \Rightarrow Y_{34}
\end{gathered}
$$

Таблица 3

Качественные показатели $K_{i}^{\prime}$ вариантов подсистемы станок $(C)$

\begin{tabular}{|c|c|c|c|c|}
\hline Подсистемы $X_{n}$ & $K_{1}^{\prime}$ & $K_{2}^{\prime}$ & $K_{3}^{\prime}$ & $K_{c}$ \\
\hline$X_{14}$ & 0,75 & 0,5 & 0,5 & 0,58 \\
\hline$X_{22}$ & 0,75 & 1,0 & 1,0 & 0,92 \\
\hline$X_{25}$ & 1,0 & 1,0 & 1,0 & 1,0 \\
\hline$X_{31}$ & 0,5 & 0,5 & 0,5 & 0,5 \\
\hline$X_{44}$ & 1,0 & 0,5 & 0,75 & 0,75 \\
\hline$X_{51}$ & 0,5 & 0,5 & 0,75 & 0,58 \\
\hline$X_{62}$ & 0,5 & 0,5 & 0,75 & 0,58 \\
\hline
\end{tabular}

Таблица 4

Качественные показатели $K_{i}^{\prime \prime}$ вариантов подсистемы приспособления (I)

\begin{tabular}{|c|c|c|c|c|}
\hline Подсистемы $Y_{m}$ & $K_{1}^{\prime \prime}$ & $K_{2}^{\prime \prime}$ & $K_{3}^{\prime \prime}$ & $K_{I I}$ \\
\hline$Y_{22}$ & 1,0 & 1,0 & 0,75 & 0,92 \\
\hline$Y_{14}$ & 1,0 & 1,0 & 1,0 & 1,0 \\
\hline$Y_{23}$ & 0,75 & 0,75 & 0,75 & 0,75 \\
\hline$Y_{24}$ & 0,75 & 0,75 & 0,75 & 0,75 \\
\hline$Y_{26}$ & 0,75 & 0,5 & 0,75 & 0,67 \\
\hline$Y_{34}$ & 1,0 & 1,0 & 0,75 & 0,92 \\
\hline
\end{tabular}

Вариант $\Pi_{l}$ имеет численное значение $K_{\Pi 1}=$ 0,84, вариант $\Pi_{2}-K_{\Pi 2}=0,90$; следовательно, второй вариант подсистем «приспособление», предназначенный для структуры участка станков $\left(C_{2}\right)$ также является более предпочтительным. 


\begin{tabular}{|c|c|c|c|c|c|c|}
\hline Impact Factor: & $\begin{array}{l}\text { ISRA (India) } \\
\text { ISI (Dubai, UAF } \\
\text { GIF (Australia) } \\
\text { JIF }\end{array}$ & $\begin{array}{l}=1.344 \\
=0.829 \\
=0.564 \\
=1.500\end{array}$ & $\begin{array}{l}\text { SIS (USA) } \\
\text { PИНЦ (Russia) } \\
\text { ESJI (KZ) } \\
\text { SJIF (Morocco) }\end{array}$ & $\begin{array}{l}=0.912 \\
=0.207 \\
=\mathbf{3 . 8 6 0} \\
=\mathbf{2 . 0 3 1}\end{array}$ & $\begin{array}{l}\text { ICV (Poland) } \\
\text { PIF (India) } \\
\text { IBI (India) }\end{array}$ & $\begin{array}{l}=6.630 \\
=1.940 \\
=4.260\end{array}$ \\
\hline
\end{tabular}

После этого выбираем по наибольшему качественному показателю $K_{i}^{\prime \prime \prime}$ подсистему инструмента на основании технических требований: стойкость (повышенная $-K_{1}^{\prime \prime \prime}=1,0$; нормативная $\left.\quad-\quad K_{1}^{\prime \prime \prime}=0,75\right)$; прочность (повышенная $\quad-\quad K_{2}^{\prime \prime \prime}=1,0 ; \quad$ соответствующая прочности стандартного инструмента $-K_{2}^{\text {"'” }}$ $=0,75) ; \quad$ регулирование геометрических параметров (автоматическое $-K_{3}^{\prime \prime \prime}=1,0$; вручную $-K_{3}^{\prime \prime \prime}=0,75$; нерегулируемые $-K_{3}^{\prime \prime \prime}=0,5$ ).

Максимальный суммарный показатель $K_{И}$ получили следующие варианты комплектов подсистемы «И»:

$$
\begin{gathered}
U_{1}=Z_{214} \Rightarrow Z_{21} \Rightarrow\left[\begin{array}{l}
Z_{11} \\
Z_{12} \\
Z_{15} \\
Z_{16} \\
Z_{18} \\
Z_{110}
\end{array}\right] \Rightarrow Z_{215} \Rightarrow Z_{31} \\
U_{2}=Z_{214} \Rightarrow Z_{21} \Rightarrow\left[\begin{array}{l}
Z_{11} \\
Z_{12}
\end{array}\right] \Rightarrow Z_{212} \Rightarrow \\
\Rightarrow Z_{31} \Rightarrow\left[\begin{array}{l}
Z_{23} \\
Z_{24} \\
Z_{25}
\end{array}\right] \Rightarrow Z_{215} \Rightarrow Z_{31}
\end{gathered}
$$

Вариант $h_{2}$ имеет численное значение $\kappa_{И}=0,71$, вариант $h_{l}$ имеет численное значение $\kappa_{И}=0,69$, следовательно второй вариант подсистемы «инструмент», предназначенный для структуры участка станков $\left(C_{2}\right)$ также является более предпочтительным.

По наибольшему качественному показателю $K_{i}^{I V}$ выбираем подсистему загрузочного устройства на основании технических требований: способ загрузки и базирования (автоматический $-K_{1}^{I V}=1,0$; механизированный $K_{1}^{I V}=0,75 ;$ ручной $\left.-K_{1}^{I V}=0,5\right)$; возможность ориентирования детали (полное ориентирование - $K_{2}^{I V}=1,0$; частичное $K_{2}^{I V}=0,75$; нет $-K_{2}^{I V}$ $=0,5$ ).

Производим выбор рациональных вариантов подсистемы «ЗУУ»

$$
\begin{gathered}
3 y_{1}=F_{11} \Rightarrow F_{11} \Rightarrow F_{21} \Rightarrow F_{21} \Rightarrow F_{21} \Rightarrow F_{12} \\
\quad ; \\
3 y_{2}=F_{11} \Rightarrow F_{11} \Rightarrow F_{11} \Rightarrow F_{12}
\end{gathered}
$$

Вариант $3 y_{l}$ имеет качественный показатель $K_{3 y_{1}}=0,87$, вариант $3 \mathrm{~V}_{2} \quad K_{3 y_{2}}=1,0$, т.е. второй вариант подсистемы «загрузочное устройство», предназначенный для структуры участка станков $\left(C_{2}\right)$ является более предпочтительным.

Далее по наибольшему качественному показателю $K_{i}^{V}$ производим выбор подсистемы транспортного устройства на основании технических требований: способ загрузки на транспортное устройство (автоматический $-K_{1}^{V}$ $=1,0 ;$ механизированный $-K_{1}^{V}=0,75$; ручной $K_{1}^{V}$ $=0,5)$; способ транспортирования детали (непрерывный $-K_{2}^{V}=1,0$; дискретный $-K_{2}^{V}$ $=0,75$; немеханизированный $-K_{2}^{V}=0,5$ ).

Производим выбор рациональных вариантов подсистемы «ТУ»

$$
\begin{gathered}
T y_{1}=H_{12} \Rightarrow H_{11} \Rightarrow H_{13} \Rightarrow H_{13} \Rightarrow H_{13} ; \\
T У_{2}=H_{12} \Rightarrow H_{11} \Rightarrow H_{13}
\end{gathered}
$$

Вариант $T У_{l}$ имеет качественный показатель $K_{T У I}=0,75$, вариант $T У_{2}-K_{T V_{2}}=0,83$.

Схемы рациональных вариантов выбранных подсистем $C_{2}, \Pi_{2}, H_{2}, 3 У_{2}, T У_{2}$ сведены в табл. 7 , на рис. 4 показана возможная компоновка ГПМ.

Качественные показатели $K_{i}^{\prime \prime \prime}$ вариантов подсистемы инструмент (И)

Таблица 5

\begin{tabular}{|c|c|c|c|c|}
\hline Подсистемы $Z_{i}$ & $K_{1}^{\prime \prime \prime}$ & $K_{2}^{\prime \prime \prime}$ & $K_{3}^{\prime \prime \prime}$ & $K_{И}$ \\
\hline$Z_{214}$ & 0,75 & 0,75 & 0,5 & 0,67 \\
\hline$Z_{21}$ & 0,75 & 0,75 & 0,5 & 0,67 \\
\hline$Z_{11}$ & 1,0 & 0,75 & 0,5 & 0,75 \\
\hline$Z_{12}$ & 1,0 & 0,75 & 0,5 & 0,75 \\
\hline$Z_{15}$ & 1,0 & 0,75 & 0,5 & 0,75 \\
\hline$Z_{16}$ & 1,0 & 0,75 & 0,5 & 0,75 \\
\hline$Z_{18}$ & 1,0 & 0,75 & 0,5 & 0,75 \\
\hline$Z_{110}$ & 0,75 & 0,75 & 0,5 & 0,67 \\
\hline
\end{tabular}

ISPC Technological development, 


\begin{tabular}{|c|c|c|c|c|c|c|}
\hline Impact Factor: & $\begin{array}{l}\text { ISRA (India) } \\
\text { ISI (Dubai, UAF } \\
\text { GIF (Australia) } \\
\text { JIF }\end{array}$ & $\begin{array}{l}=1.344 \\
=0.829 \\
=0.564 \\
=1.500\end{array}$ & $\begin{array}{l}\text { SIS (USA) } \\
\text { PИНЦ (Russia) } \\
\text { ESJI (KZ) } \\
\text { SJIF (Morocco) }\end{array}$ & $\begin{array}{l}=0.912 \\
=0.207 \\
=\mathbf{3 . 8 6 0} \\
=\mathbf{2 . 0 3 1}\end{array}$ & $\begin{array}{l}\text { ICV (Poland) } \\
\text { PIF (India) } \\
\text { IBI (India) }\end{array}$ & $\begin{array}{l}=6.630 \\
=1.940 \\
=4.260\end{array}$ \\
\hline
\end{tabular}

\begin{tabular}{|c|c|c|c|c|}
\hline Подсистемы $Z_{i}$ & $K_{1}^{\prime \prime \prime}$ & $K_{2}^{\prime \prime \prime}$ & $K_{3}^{\prime \prime \prime}$ & $K_{И}$ \\
\hline$Z_{212}$ & 0,75 & 0,75 & 0,5 & 0,67 \\
\hline$Z_{215}$ & 0,75 & 0,75 & 0,5 & 0,67 \\
\hline$Z_{23}$ & 0,75 & 0,75 & 0,5 & 0,67 \\
\hline$Z_{24}$ & 0,75 & 0,75 & 0,5 & 0,67 \\
\hline$Z_{29}$ & 0,75 & 0,75 & 0,5 & 0,67 \\
\hline$Z_{231}$ & 0,75 & 0,75 & 0,5 & 0,67 \\
\hline
\end{tabular}

Таблица 6

Качественные показатели $K_{i}^{\prime \prime \prime}$ вариантов подсистемы загрузочное устройство (ЗУ)

\begin{tabular}{|c|c|c|c|}
\hline Подсистемы $F_{i}$ & $K_{1}^{I V}$ & $K_{2}^{I V}$ & $K_{3 у}$ \\
\hline$F_{11}$ & 1,0 & 1,0 & 1,0 \\
\hline$F_{12}$ & 1,0 & 1,0 & 1,0 \\
\hline$F_{21}$ & 0,75 & 0,75 & 0,75 \\
\hline
\end{tabular}

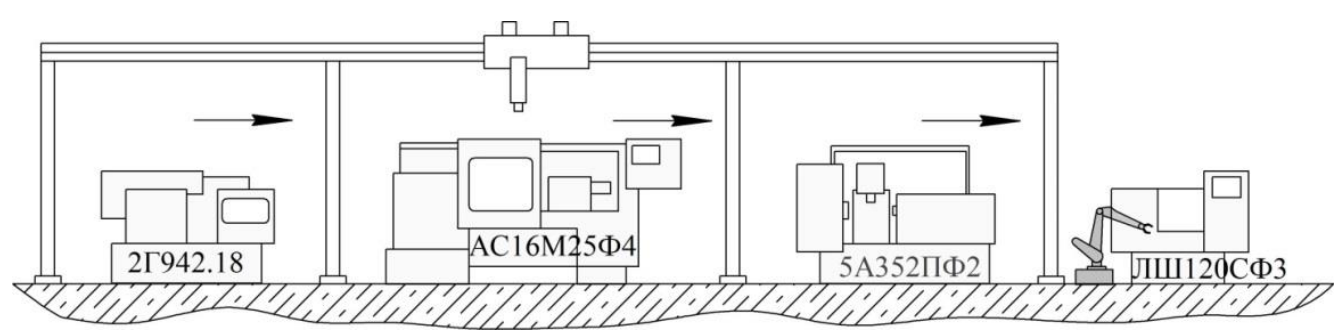

Рисунок 4 - Компоновка ГПМ

Таблица 7

Синтезированный структурный вариант ГПМ

\begin{tabular}{|c|c|}
\hline Обозначение & Структура подсистемы \\
\hline$C_{2}$ & $X_{14} \Rightarrow X_{25} \Rightarrow X_{51} \Rightarrow X_{62}$ \\
\hline$\Pi_{2}$ & $Y_{22} \Rightarrow Y_{14} \Rightarrow Y_{24} \Rightarrow Y_{34}$ \\
\hline$H_{2}$ & $Z_{214} \Rightarrow Z_{21} \Rightarrow\left[\begin{array}{c}Z_{11} \\
Z_{12}\end{array}\right] \Rightarrow Z_{212} \Rightarrow Z_{31} \Rightarrow Z_{21} \Rightarrow\left[\begin{array}{c}Z_{23} \\
Z_{24} \\
Z_{25}\end{array}\right] \Rightarrow Z_{215} \Rightarrow Z_{31}$ \\
\hline $3 Y_{2}$ & $F_{11} \Rightarrow F_{11} \Rightarrow F_{11} \Rightarrow F_{12}$ \\
\hline$T Y_{2}$ & $H_{12} \Rightarrow H_{11} \Rightarrow H_{13}$ \\
\hline
\end{tabular}

\section{Заключение}

Проведенный анализ позволил выявить из сформированного множества вариантов подсистем автоматизированных участков ремонтного производства для изготовления деталей типа тела вращения несколько рациональных структур, среди которых одна $\left(C_{2}\right)$ имеет более высокие качественные показатели.

Для более полной информации и объективной оценки анализируемых вариантов необходим учет стохастических процессов, происходящих в технологической системе каждого ГПМ в реальных условиях функционирования на основе структурно параметрической оптимизации. Этап требует проведения дополнительных теоретических и практических исследований реальных конструкций подсистем ГПМ, которые приведут к реализации наиболее эффективной структуры участка. 


\begin{tabular}{l|lrl|l|ll} 
& ISRA (India) & $=\mathbf{1 . 3 4 4}$ & SIS (USA) & $=\mathbf{0 . 9 1 2}$ & ICV (Poland) & $=\mathbf{6 . 6 3 0}$ \\
Impact Factor: & ISI (Dubai, UAE) $=\mathbf{0 . 8 2 9}$ & PUHU (Russia) $=\mathbf{0 . 2 0 7}$ & PIF (India) & $=\mathbf{1 . 9 4 0}$ \\
& GIF (Australia) & $\mathbf{0 . 5 6 4}$ & ESJI (KZ) & $=\mathbf{3 . 8 6 0}$ & IBI (India) & $\mathbf{4 . 2 6 0}$
\end{tabular}

\section{References:}

1. (2004) Avtomatizacija proizvodstvennyh processov $\mathrm{v}$ mashinostroenii: Ucheb. dlja vtuzov / N.M. Kapustin, P.M. Kuznecov, A.G. Shirtladze i dr.; Pod red. N.M. Kapustina. - M.: Vyssh. shk., 2004. - 415 p.

2. Raluca Nicolae (2015) Flexibility and efficiency analysis of a flexible manufacturing system/ Raluca Nicolae, Anisor Nedelcu// Review of the Air Force Academy No 1 (28) 2015. - PP.155-158.

3. M. Carmen Ruiz (2009) Improving performance in flexible manufacturing systems/M. Carmen Ruiz [at all] //The Journal of Logic and Algebraic Programming, 78 (2009). - PP.260-273.

4. Grega V.M. (2010) Gibkost' proizvodstva kak faktor obespechenija konkurentosposobnosti produkcii avtomobilestroenija/V.M. Grega//Jekonomicheskie nauki - 3(64), 2010. PP..194-196.

5. Hermaste A. (2014) Design principles of flexible manufacturing systems / A. Hermaste, J. Riives, K. Sonk, M. Sarkans. // 9th International DAAAM Baltic Conference «INDUSTRIAL ENGINEERING» 24-26 April 2014, Tallinn, Estonia. - PP.92-96.

6. Mjamlin V.V. (2013) Gibkie potoki dlja remonta vagonov i osobennosti imitacionnogo modelirovanija ih raboty/ V.V. Mjamlin// Transport Rossijskoj Federacii, № 3 (46) 2013. - p.57-60.

7. Epifanov V.V. (2005) Razrabotka tehnologicheskih struktur metallorezhushhego oborudovanija s programmnym upravleniem dlja gruppovoj obrabotki slozhnyh detalej vrashhenija. Diss. dokt.tehn. nauk. Ul'janovsk, 2005. 323p.

8. Puhov A.S. (2009) Sintez reshenij pri poiskovom proektirovanii avtomatizirovannyh sistem: Monografija. - Kurgan: Izd-vo Kurganskogo gos. un-ta, 2009.- 154 p.

9. Tiberiu S. Letia. (2012) Analysis and Synthesis of the Flexible Manufacturing System Potential Behaviors/ Tiberiu S. Letia, Adina Astilean, Mihai Hulea//Proceedings of the 14th IFAC Symposium on Information Control Problems in Manufacturing Bucharest, Romania, May 2325, 2012. - PP.248-253.

10. Bogutsky V.B. (2017) Sistemnyj analiz i strukturnyj sintez mehanoobrabatyvajushhih modulej dlja remontnogo proizvodstva / VB. Boguckij, N.V Popravka//Aktual'nye voprosy v nauke i praktike. Sbornik statej po materialam III mezhdunarodnoj nauchno-prakticheskoj konferencii (g. Kazan'). V 4 ch. Ch.1 / - Ufa: Izd. Dendra, 2017. - PP. 130-136.

11. Kudrjashov E.A. (2009) Primenenie gruppovogo metoda $\mathrm{v}$ organizacii remontnogo proizvodstva/ E.A. Kudrjashov, A.V. Stecurin // Izvestija Kurskogo gosudarstvennogo tehnicheskogo universiteta. 2009. № 1 (26). PP.8-12.

12. Nikola Suzić. (2012) Customizing Products through Application of Group Technology: A Case Study of Furniture Manufacturing/ Nikola Suzić, Branislav Stevanov, Ilija Ćosić, Zoran Anišić, Nemanja Sremčev // Journal of Mechanical Engineering 58(2012)12, - PP. 724-731. DOI:10.5545/sv-jme.2012. 708. 